\title{
The MAGEC System for Spinal Lengthening in Children with Scoliosis: A NICE Medical Technology Guidance
}

\author{
Michelle Jenks $\cdot$ Joyce Craig $\cdot$ Joanne Higgins $\cdot$ \\ Iain Willits · Teresa Barata • Hannah Wood • \\ Christine Kimpton • Andrew Sims
}

Published online: 30 August 2014

(c) The Author(s) 2014. This article is published with open access at Springerlink.com

\begin{abstract}
Scoliosis-structural lateral curvature of the spine - affects around four children per 1,000. The MAGEC system comprises a magnetically distractible spinal rod implant and an external remote controller, which lengthens the rod; this system avoids repeated surgical lengthening. Rod implants brace the spine internally and are lengthened as the child grows, preventing worsening of scoliosis and delaying the need for spinal fusion. The Medical Technologies Advisory Committee at the National Institute for Health and Care Excellence (NICE) selected the MAGEC system for evaluation in a NICE medical technologies guidance. Six studies were identified by the sponsor (Ellipse Technologies Inc.) as being relevant to the decision problem. Meta-analysis was used to compare the clinical evidence results with those of one conventional growth rod study, and equal efficacy of the two devices was concluded. The key weakness was selection of a single comparator study. The External Assessment Centre (EAC) identified 16 conventional growth rod studies and undertook meta-analyses of relevant outcomes. Its critique highlighted limitations around study heterogeneity and variations in baseline characteristics and follow-up duration,
\end{abstract}

M. Jenks $(\bowtie) \cdot$ J. Craig $\cdot$ H. Wood

York Health Economics Consortium, University of York,

Level 2, Market Square, York YO10 5NH, UK

e-mail: michelle.jenks@york.ac.uk

\section{J. Higgins}

National Institute for Health and Care Excellence, Level 1A,

City Tower, Piccadilly Plaza, Manchester M1 4BT, UK

I. Willits · C. Kimpton · A. Sims

Newcastle upon Tyne Hospitals NHS Foundation Trust, Freeman

Hospital, Freeman Road, Newcastle upon Tyne NE7 7DN, UK

T. Barata

Quantics Consulting Ltd, West End House, 28 Drumsheugh

Gardens, Edinburgh EH3 7RN, UK precluding the ability to draw firm conclusions. The sponsor constructed a de novo costing model showing that MAGEC rods generated cost savings of $£ 9,946$ per patient after 6 years, compared with conventional rods. The EAC critiqued and updated the model structure and inputs, calculating robust cost savings of $£ 12,077$ per patient with MAGEC rods compared with conventional rods over 6 years. The year of valuation was 2012. NICE issued a positive recommendation as supported by the evidence (Medical Technologies Guidance 18).

\section{Key Points for Decision Makers}

The case for adopting the MAGEC system for spinal lengthening in children with scoliosis is supported by the evidence. Use of the MAGEC system would avoid repeated surgical procedures for growth rod lengthening. This could reduce complications and could have other physical and psychological benefits for affected children and their families.

The MAGEC system is indicated for use in children aged 2 years and over with scoliosis who need surgery to correct their spinal curvature-for example, when conservative methods such as bracing or casting have failed.

Findings from cost modelling estimate that use of the MAGEC system is cost saving, compared with conventional growth rods, from about 3 years after the first insertion. The estimated cost saving per child after 6 years is around $£ 12,077$. The cost savings remained robust in sensitivity analyses. Further savings could be made by avoiding the need for spinal cord monitoring, which is sometimes used during conventional growth rod lengthening but is not needed for lengthening of MAGEC growth rods. 


\section{Introduction}

The National Institute of Health and Care Excellence (NICE) produces evidence-based medical technologies guidance with the overall aims of evaluating and, where appropriate, encouraging adoption of novel and innovative medical devices and diagnostics within the National Health Service (NHS) in England. Manufacturers or distributors of potentially eligible technologies submit notifications regarding their products to NICE's Medical Technologies Evaluation Programme (MTEP). Technologies are selected for evaluation by the programme if they have the potential to offer significant clinical benefits to patients and the NHS or to reduce costs, compared with current standard practice. Guidance is issued after the clinical and cost evidence submitted by the sponsor is assessed independently by an External Assessment Centre (EAC) and after a public consultation period. Devices and diagnostic tools with more complex value propositions can be routed for evaluation through other NICE programmes, such as the Diagnostics Assessment Programme. Campbell and Campbell [1] have described the methods of MTEP in more detail.

The MAGEC system comprises a magnetically distractible spinal rod implant and an external remote controller, which is used to distract (lengthen) the implant. The device, developed by the sponsor Ellipse Technologies Inc., is used to prevent worsening of scoliosis in children who have not responded to other treatments. The EAC critiquing the evidence was the Newcastle upon Tyne Hospitals NHS Foundation Trust and York Health Economics Consortium partnership. NICE published its final guidance on this device in June 2014.

This article provides a summary of the sponsor's submission on the device's clinical and cost effectiveness, and an overview of the EAC's outputs and subsequent development of the NICE guidance. Full documentation of the process, supporting evidence and the final guidance can be found on the NICE website [2].

\section{Background to the Condition and Device}

Scoliosis is a structural lateral curvature of the spine, which occurs most commonly in children and young adolescents [3]. Scoliosis may result from other medical conditions but more commonly has no known cause (idiopathic scoliosis) [4]. In the majority of cases, treatment is not required and the mild condition corrects itself as the child grows. Children with severe and progressive disease, if left untreated, are at risk of rapid spinal deformity progression, cosmetic disfigurement and pulmonary insufficiency.
Untreated scoliosis can also reduce life expectancy, because of respiratory failure. This problem is exacerbated in children who develop scoliosis at a very young age [3].

Various options exist for children who require treatment for scoliosis, and, as no standardized treatment guidelines exist within England, methods differ between clinicians and hospitals. Following diagnosis, most children will either be observed, wear a back brace [3] or wear a cast [5]. These preventative methods aim to stop the curve from worsening as the child grows, rather than cure the scoliosis [6]. Surgery may be required in children for whom the condition progresses. Spinal fusion was advocated in the past for children with severe curves or progression despite bracing; however, fusion can lead to arrested pulmonary development and thoracic insufficiency syndrome (inability of the thorax to support normal respiration and lung growth) [3]. Growth rods were developed to limit progression of the condition, improving thoracic volume as the child grows, whilst delaying the need for spinal fusion [7].

Growth rods are distractible spinal implants, which are attached to the spine, using hooks and pedicle screws, under general anaesthetic. A single rod can be attached on one side of the curve, or, more commonly, rods are attached to both sides of the spine (dual rods). The rods brace the spine internally and are distracted as the child grows, to control the progression of the spinal deformity [8]. Conventional growth rods are lengthened every 6 months during an invasive procedure in which the surgeon re-opens the surgical incision site and distracts the rods under general anaesthetic [7].

MAGEC rods are inserted in the same way as conventional growth rods; however, they are distracted magnetically, using an external remote controller in a noninvasive procedure during an outpatient appointment. The sponsor claimed that avoidance of repeated surgeries reduced the incidence of surgical complications, reduced psychological trauma and improved patients' quality of life. The costs associated with repeated surgical interventions would also be avoided. The MAGEC system was considered by the Medical Technologies Advisory Committee (MTAC) as an alternative to conventional growth rods in children with scoliosis, as defined in the scope produced by NICE. This is described further in the following section.

\section{Decision Problem (Scope)}

\subsection{Population}

The population described in the scope was children with scoliosis in whom a surgical distraction procedure was indicated. This population included children who had either idiopathic or symptomatic scoliosis and, in many cases, 
had failed on more conservative treatment methods. The MAGEC system is contraindicated for children under the age of 2 years.

\subsection{Comparator (Current Practice)}

Spine-based surgical distraction procedures with conventional growth rods were specified as the comparator within the scope. Other techniques that have been used to correct childhood scoliosis, including the Shilla growth guidance technique and the Luqué trolley construct, were judged as being out of scope for the decision problem, as these techniques were understood not to be current standard practice in the NHS in England. Both single and dual conventional rod constructs were relevant.

\subsection{Intervention (the MAGEC System)}

The intervention outlined in the scope was the MAGEC system, with single or dual rod constructs and an external remote controller. Adjustments can be made with the external remote controller to lengthen or shorten the rod, if necessary. The growth rods are available in two diameters (4.5 and $5.5 \mathrm{~mm}$ ); the recommended size depends upon the bodyweight of the child and the discretion of the surgeon.

\subsection{Outcomes}

Twelve outcomes were listed in the scope as being relevant to the decision problem, and five of these were formally addressed within the sponsor's submission. The sponsor considered a further seven intermediate outcome measures, which were not identified in the scope (Table 1). The sponsor's included outcomes were those consistently reported in published clinical literature, including the Cobb angle, thoracic spine height and total spine height.

The Cobb angle is the standard method used to quantify spinal curvature. It measures the angle between the endplates of the end vertebrae of the scoliotic curve measured on a radiograph of the coronal (frontal) plane [9].

The thoracic spine region, containing 12 vertebrae (the $\mathrm{T} 1-\mathrm{T} 12$ vertebrae), is the largest region of the spine. This region of the spine protects the thoracic viscera-for example, the heart and lungs [10]. Carrying out spinal fusion in children with a thoracic spine height below normal height can increase the risk of restrictive lung disease and the 'crank shaft phenomenon', whereby there is continued growth in the anterior of the spine when it is fused from the posterior. Growth rods may enable children with scoliosis to achieve an adequate thoracic spine height prior to spinal fusion [11]. Therefore, thoracic spine height is measured as an outcome in most growth rod studies and was included in the sponsor's analysis.
The total spine height (the T1-S1 vertebrae) encompasses the thoracic spine region and the lumbar and sacral vertebrae lower on the spine. The reasons why it is important for children with scoliosis to achieve adequate total spine height include those listed for the thoracic spine height (because of inclusion of the thoracic spine region) and cosmetic reasons. This measure is also used in most clinical studies of growth rods.

\section{Review of Clinical and Economic Evidence}

Clinical and economic evidence were submitted by the sponsor, in line with the process set out by NICE [12]. The sponsor submitted a de novo costing model as part of its economic evidence. The EAC critically appraised the submission and the costing model. Section 4.1 summarizes the clinical evidence submitted, the EAC critique and the EAC's new work. Section 4.2 provides the same detail for the economic evidence.

\subsection{Clinical Effectiveness Evidence}

\subsubsection{Sponsor's Review of Clinical Effectiveness Evidence}

The sponsor undertook a search for published clinical evidence relating to the decision problem, which identified four studies suitable for inclusion [7, 13-15]. A further four unpublished studies were identified and were included by the sponsor [16-19]. One of these four studies has subsequently been published [18]. Two studies were judged as being out of scope-one being an animal study [15] and the other being a cost analysis [17] (which was subsequently considered as economic evidence).

Four of the six studies were prospective case series [7, $13,14,18]$, with one being a retrospective case review [16] and one being a retrospective case-matched series of 12 patients with MAGEC rods and 12 with conventional growth rods [19]. Both retrospective reviews drew on patients from several centres; details on processes such as follow-up and measurement were not provided. The mean follow-up period ranged from 10 months to 2.5 years, with a weighted mean, using patient numbers as weights, of 19 months. All studies recruited patients with childhood scoliosis, except for one study [16], which included a wider patient group-children with any early-onset spinal deformity.

There was some overlap of patients within the included studies, with one study [14] including all of the patients from another [18]; the latter is not discussed further in this article. Twelve patients receiving a MAGEC rod in the case-matched series [19] were included in the case review [16]. The results of the case-matched study [19] are 
Table 1 Outcomes in scope and sponsor's submission

\begin{tabular}{|c|c|}
\hline Outcomes to consider in scope issued by NICE & Outcomes considered in sponsor's submission \\
\hline $\begin{array}{l}\text {-Total numbers of surgical procedures } \\
\text { and anaesthetics } \\
\text {-Total numbers of outpatient attendances and procedures } \\
\text { - Recovery time } \\
\text { - Total length of stay } \\
\text { - Rate of distraction procedure success } \\
\text {-Infection rates and other surgical } \\
\text { complication rates } \\
\text {-Total number of imaging procedures } \\
\text {-Quality of life } \\
\text {-Device failure } \\
\text {-Device and radiation exposure-related } \\
\text { adverse events }\end{array}$ & $\begin{array}{l}\text { Suggested in scope } \\
\text {-Total number of surgical procedures } \\
\text { - Total number of outpatient procedures } \\
\text { - Rate of distraction procedure success } \\
\text { - Infection rates and other surgical complication } \\
\text { rates } \\
\text {-Device failure } \\
\text { Variation from scope } \\
\text {-Cobb angle } \\
\text {-Thoracic spine height (T1-T12) } \\
\text {-Total spine height (T1-S1) } \\
\text {-Length of stay (not quantified) } \\
\text {-Forced volume vital capacity } \\
\text { - Forced expiratory volume } \\
\text {-Thoracic kyphosis }\end{array}$ \\
\hline
\end{tabular}

NICE National Institute for Health and Care Excellence
The change in the thoracic spine height ${ }^{1}$ of $31 \mathrm{~mm}$ in the MAGEC system studies [7, 13, 14, 16] was not compared with the changes reported in conventional rod studies, because of data limitations. Compared with the findings in the conventional rod study [20], the infection rates $(15 \%$ versus $6.4 \%)$, numbers of surgical procedures (6.4 versus 1.1) and numbers of device failures per patient (0.37 versus 0.07$)$ were lower in the MAGEC rod studies $[7,13,14,16]$.

The sponsor concluded that the clinical evidence demonstrated that MAGEC rods are equally as effective as conventional rods and provide a reduced risk of adverse events.

\subsubsection{Critique of Clinical Effectiveness Evidence}

The EAC critiqued the literature search, evidence selection and quality assessment of the included studies completed by the sponsor, and consulted clinical experts, identified using NICE's published processes, for advice on relevant comparators and patient groups. Where possible, the EAC repeated the sponsor's searches and obtained a broadly similar yield of search results. The study selection criteria employed by the sponsor limited the intervention to the MAGEC system and placed no constraints on outcomes, study design, language or search dates. It was unclear how the sponsor had selected the comparator study [20].

The EAC reviewed each paper for potential bias, limitations, inconsistencies in results and uncertainty around generalizability. Each study was graded; all were case series, which scored 3 on the Scottish Intercollegiate Guidelines Network (SIGN) grading [21] and 'very low' on the Grading of Recommendations Assessment, Development and Evaluation (GRADE) [22], thus any estimate of
${ }^{1}$ The changes in the Cobb angle, total spine height and thoracic spine height were calculated from the random-effects model. The difference between final follow-up results and pre-operative results were used. 


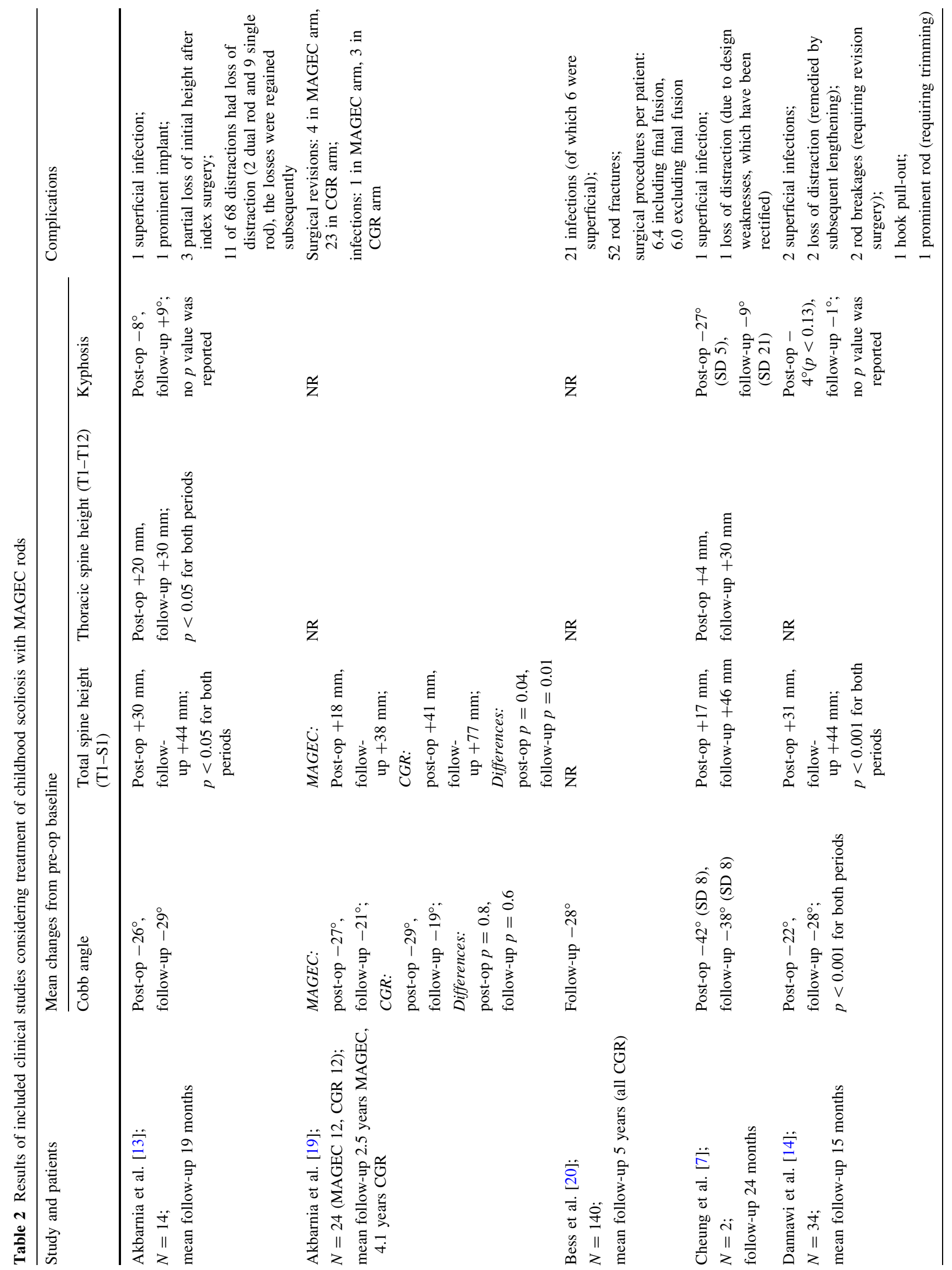




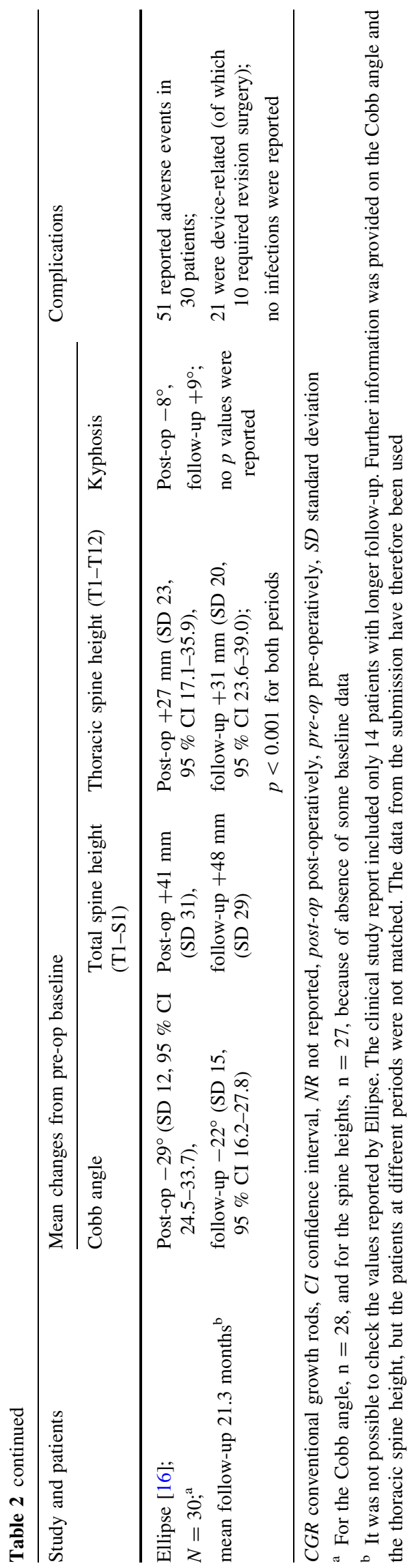

the effect is very uncertain. Meta-analysis could not mitigate fully against this underlying uncertainty. Case series are prone to selection and observer bias when patients are selected for inclusion in the study and in reporting outcomes. The reporting of inclusion and exclusion criteria within the included studies was generally poor, with two studies not reporting inclusion criteria $[7,18]$ and only one study reporting any exclusion criteria [14]. The sponsor failed to provide discussion around limitations relating to the included studies.

The technique used by the sponsor for meta-analysis was not reported; only the results were presented. It was thus not possible to critique the methodology and hence to have confidence in the results. The largest weaknesses identified following the critique were the absence of a rationale for the choice of the comparative study, and the lack of discussion of differences in follow-up durations between the conventional and MAGEC rod studies and their potential implications for comparison of outcomes. Given these limitations within the sponsor's submission, the EAC undertook additional work.

The EAC undertook a literature search to identify any MAGEC rod studies that had not been identified from the sponsor's search and to identify studies of conventional growth rods to inform the comparator. A number of databases (including Medline, the Cochrane Library and Embase) and the grey literature were searched (the strategies that were adopted are available from the EAC assessment report [2]).

The included studies of conventional rods were limited to those that included at least 20 patients and were published after 1 January 2008. The choice of 20 patients was informed by a scoping search: a cut-off was required pragmatically to enable the EAC to manage the studies within the evaluation timescale. The 2008 filter was applied to capture studies of current growth rod technologies and hence appropriate comparators for MAGEC rods.

The Preferred Reporting Items for Systematic Reviews and Meta-Analyses (PRISMA) diagram [23] (Fig. 1) shows the numbers of papers that were retrieved and excluded following dual selection by two independent reviewers, together with the reasons for exclusion. Of the 23 included studies, 15 [20,24-37] were studies of conventional rods and eight were studies of MAGEC rods [7, 13, 14, 16, 18, 19, 38, 39]. Of the eight studies of MAGEC rods, two were additional to those found from running the sponsor's search [38, 39]; however, the sponsor advised that patients in these studies were already included in another study [14]. The conventional rod arm of the case-matched study [19] included by the sponsor was also included within the evidence base, totalling 16 studies. Of the 16 conventional rod studies, 15 were retrospective case series and the remaining one was a prospective case series [28]. 
Data were extracted from the included studies, and the weighted mean follow-up duration was calculated, using the number of patients within each study as a weight. The extraction was undertaken by one reviewer and checked by a second reviewer. There were notable differences between the conventional rod studies and the MAGEC rod studies. The latter included a greater mean Cobb angle at baseline $\left(72.4^{\circ}\right.$ versus $\left.65.7^{\circ}\right)$; a lesser mean total spine height at baseline (258 versus $288 \mathrm{~mm}$ ) and a younger mean age at surgery (6.4 versus 8.0 years). These differences were likely to influence the potential changes in each outcome over time. For example, a greater height at baseline may limit the potential for growth during follow-up periods; similarly, a less severe Cobb angle may limit the potential improvement in that outcome. The mean duration of follow-up in the conventional rod studies was 4.3 years (range 2.5-6.6 years). This was materially longer than the followup in any of the MAGEC system studies (range 10 months to 2.5 years). The number of distractions is a function of time, and these are related to other outcomes of interest, including the change in the Cobb angle, the change in spine height and the change in infection rates. Hence the difference in the follow-up durations was a major source of confounding and limited the ability to use the evidence to inform recommendations.

Meta-analyses were undertaken by the EAC for the MAGEC rod and conventional rod studies, following data extraction into a predefined template. The data items that were required included changes in mean differences in the Cobb angle and spine height, and a measure of variance. Not all studies provided usable data. Given the variation in the duration of follow-up between the two sets of studies, the studies were grouped into those with less than, or greater than, 38 months of follow-up. Those conventional rod studies with a follow-up period of less than 38 months were considered to be more comparable to the MAGEC rod studies. The few ( $n=3$ to $n=8$ ) studies of conventional rods that provided sufficient statistical data to enable them to be included in the longer-term follow-up analyses is an indication of how poor the reporting of many of these studies was. Variance, ranges and CIs were particularly poorly reported. The MAGEC rod studies reported results better than the majority of the conventional rod studies. This variation in reporting could be explained in part by the prospective nature of four of the six MAGEC rod studies $[7,13,14,18]$, compared with the retrospective nature of all but one [28] of the conventional rod studies.

The included studies and their results are shown in Table 3. Fixed- and random-effects models were fitted. Heterogeneity was assessed by using the $I^{2}$ statistic, which measures the percentage of variation across studies that is due to heterogeneity rather than sampling error. Benchmarks were suggested for $I^{2}$ : values of the order of $25 \%$

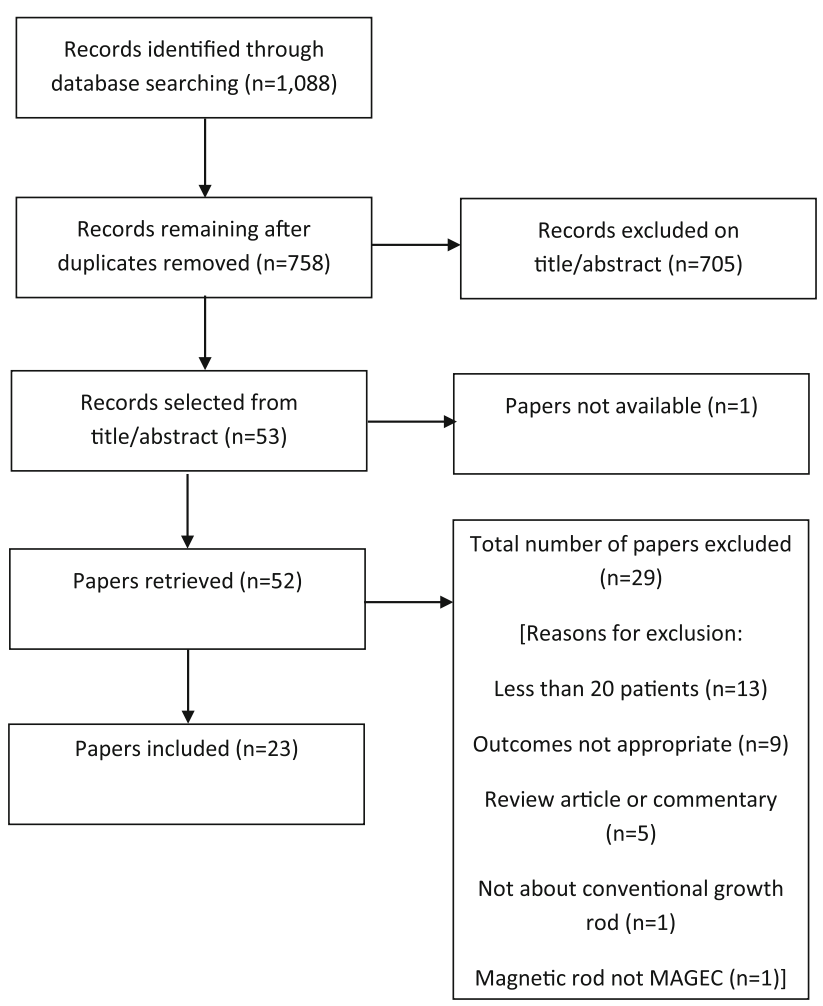

Fig. 1 Preferred Reporting Items for Systematic Reviews and MetaAnalyses (PRISMA) flow diagram for External Assessment Centre (EAC) clinical evidence review

were considered low, $50 \%$ moderate and $75 \%$ high [40]. Where studies were heterogeneous, the random-effects model was more appropriate because (unlike the fixedeffects model) it incorporates the heterogeneity among studies. The results for meta-analysis with low heterogeneity scores tend to be similar for the fixed- and randomeffects models.

The mean differences in the Cobb angle between baseline and follow-up were $27^{\circ}$ for the MAGEC rod group and $32^{\circ}$ for the longer follow-up conventional rod group (CI reported in Table 3). However because of the differences in follow-up periods, the meta-analysis results for these two groups were unlikely to be comparable. Only one study had sufficient data to be included in the shorter follow-up group [37], and there was considerable variation between this and the conventional growth rod studies with longer follow-up. For the total spine height, the estimated mean difference between baseline and follow-up was $4.55 \mathrm{~cm}$ with MAGEC rods and $10.76 \mathrm{~cm}$ with conventional rods (in the shorter follow-up group). The very high heterogeneity between the conventional rod studies greatly limits the confidence of those results. Pfandlsteiner et al. [29] reported the highest value for changes in the total spine height of all of the studies. The patients in their study tended to be older at the time of surgery than those in the 
Table 3 Results of the External Assessment Centre (EAC) meta-analysis

\begin{tabular}{|c|c|c|c|c|}
\hline \multirow[t]{2}{*}{ Parameter } & \multirow[t]{2}{*}{ Number of studies included } & \multirow{2}{*}{$\begin{array}{l}\text { Heterogeneity } \\
\left(I^{2} \text { statistic) }\right.\end{array}$} & \multicolumn{2}{|l|}{ Mean $(95 \%$ CI $)$} \\
\hline & & & Fixed-effects model & Random-effects model \\
\hline \multicolumn{5}{|l|}{ Cobb angle } \\
\hline MAGEC rods & $4[7,13,14,16]$ & 44.89 & $27.16^{\circ}(24.41-29.92)$ & $27.17^{\circ}(23.12-31.22)$ \\
\hline \multicolumn{5}{|l|}{ Conventional rods } \\
\hline Shorter follow-up & $1[37]$ & NA (1 study) & $37.03^{\circ}(27.26-46.80)$ & - \\
\hline Longer follow-up & $4[25,27,30,32]$ & 34.83 & $32.14^{\circ}(28.91-35.36)$ & $32.90^{\circ}(28.61-37.18)$ \\
\hline \multicolumn{5}{|c|}{ Change in total spine height } \\
\hline MAGEC rods & $4[7,13,14,16]$ & 0 & $4.55 \mathrm{~cm}(3.98-5.11)$ & $4.55 \mathrm{~cm}(3.98-5.11)$ \\
\hline \multicolumn{5}{|l|}{ Conventional rods } \\
\hline Shorter follow-up & $2[29,37]$ & 92.33 & $12.29 \mathrm{~cm}(11.16-13.43)$ & $10.76 \mathrm{~cm}(5.53-15.98)$ \\
\hline Longer follow-up & $3[25,27,30]$ & 96.5 & $4.25 \mathrm{~cm}(3.77-4.72)$ & $6.43 \mathrm{~cm}(2.70-10.15)$ \\
\hline \multicolumn{5}{|c|}{ Infection rates (per patient) } \\
\hline MAGEC rods & $4[7,13,14,16]$ & 61.68 & $0.03(0.00-0.08)$ & $0.04(0.00-0.15)$ \\
\hline \multicolumn{5}{|l|}{ Conventional rods } \\
\hline Shorter follow-up & $2[33,37]$ & 83.65 & $0.03(0.00-0.08)$ & $0.03(0.00-0.25)$ \\
\hline Longer follow-up & $8[20,24,25,27,28,31,32,35]$ & 57.33 & $0.14(0.11-0.16)$ & $0.15(0.11-0.20)$ \\
\hline
\end{tabular}

CI confidence interval, $N A$ not applicable

other studies. If that study is excluded, the results for this group are based on the study by Wang et al. [37] only, for which a mean change in the total spine height of $7.96 \mathrm{~cm}$ was reported. The average number of infection episodes per patient with MAGEC rods was 0.03-0.04. For the shorter follow-up conventional rod group, the estimated average number of infection episodes per patient was about 0.03 , and heterogeneity was quite high. Only two studies had enough data to be included in the meta-analysis. Wang et al. [37] only reported on deep wounds and not both deep and superficial wounds, as was the case for the rest of the studies. If that study is excluded, the results for this group are based on the study by Zhao et al. [33], who reported an average number of infection episodes per patient of 0.12 . The average number of infection episodes per patient in the longer follow-up group was estimated to be around $0.14-0.15$.

Quantitative analysis was carried out around device failure (limited to rod breakage, rather than either failure to distract or screw failure), the rate of surgical procedures and the rate of distraction. The mean number of distractions per year was greater for the MAGEC rod studies (4.5) than for all conventional rod studies (1.1). The mean number of surgeries per patient per year was smaller in the MAGEC studies (0.9) than in the conventional rod studies (1.5 for shorter follow-up and 1.1 for longer follow-up). The mean rates of device failure per child were lower in the MAGEC group than in either conventional growth rod group (MAGEC studies $6 \%$, shorter follow-up conventional rod studies $13 \%$, longer follow-up conventional rod studies $31 \%$ ); however, the annualized rates were similar at around $4.5 \%$ in the MAGEC rod studies and in the shorter follow-up conventional rod studies, with the longer follow-up studies having an annualized rate of $7.2 \%$.

Information on the impact of the procedure on quality of life was reported in only one study [7]. No evidence was reported on ability to conduct daily activities, school attendance or psychological trauma. The sponsor claimed that use of the MAGEC system would improve these aspects, compared with conventional growth rods. Clinical experts advised the EAC and MTAC of the psychological trauma that some children with conventional growth rods experience as a result of the need for repeated surgeries. In some instances, children have developed 'hospital phobia' and refused any further distractions. As the use of the MAGEC system overcomes the need for repeated surgery, limiting benefit to the clinical endpoints may understate the value that patients place on the improvement they experience.

The results from the meta-analysis were subject to limitations arising from the high heterogeneity existing across the conventional rod studies; the confounding factor of differences in the baseline characteristics of patients in the MAGEC and conventional rod studies (relating to the total spine height, age and Cobb angle), fundamental differences in the study designs and the substantially shorter follow-up duration reported in the MAGEC rod studies. The EAC judged that there was neither the quality nor the quantity of evidence to draw firm conclusions around the clinical efficacy of MAGEC rods compared with conventional rods and, as such, MAGEC rod studies with longer follow-up durations are required. From a patient 
perspective, the absolute number of surgeries is the key driver. It was apparent from the clinical evidence that this number was reduced with MAGEC rods compared with conventional rods.

\subsection{Economic Evidence}

\subsubsection{Sponsor's Economic Submission}

The sponsor undertook a limited search for economic evidence, which was based upon the strategy reported by Richards and Nnadi in their cost study [17]. No other economic studies were identified, hence that study [17] was the only economic study to be included. Richards and Nnadi collected data from 14 children undergoing growth rod surgery for scoliosis. The included patients either underwent primary surgery (MAGEC insertion) or conversion surgery (replacement of conventional rods with MAGEC rods). A unit cost analysis was undertaken to compare the costs of conventional growth rod treatment ( $n=8$ children) and MAGEC rod treatment $(n=14$ children). The authors used the data that were collected and the costs that were calculated to forecast cost savings over a 10-year time horizon [17].

A de novo temporal cost minimization model was submitted by the sponsor and compared the MAGEC system and conventional growth rods over a 6-year time horizon from a NHS and Personal Social Services perspective, with the year of valuation being 2012. The model was created in Statistical Analysis Software ${ }^{\circledR}$, and the results were copied into Microsoft Excel ${ }^{\circledR}$ for submission. The structure of the model was identical for both the treatment and the comparator. In month 0 , the cost of insertion of the rods, plus the cost of complete device failure multiplied by the incidence of this failure, were included. In subsequent months, the cost of each lengthening was added, and the total cumulative cost after 6 years was calculated for both the MAGEC and conventional rod arms of the model.

The costs of rod insertion, rod lengthening and device failure for MAGEC and conventional rods were obtained from Richards and Nnadi [17]. Other model inputs were the frequency of rod lengthening and the rate of device failure. Within the sponsor's model, the cost of distraction of MAGEC rods was applied every 3 months, and that of conventional rod lengthening was applied every 6 months. In the base case, a device failure rate of $0 \%$ was assumed.

The results were reported as the month in which breakeven occurred between the devices, absolute costs (analysed by cost category) and cost differences at 6 years. The base case results from the model showed that MAGEC rods generated cost savings of $£ 9,946$ per patient after 6 years, compared with conventional rods. At 6 years, the cumulative per-patient cost of MAGEC rods was $£ 36,094$ versus $£ 46,040$ for conventional rods. Breakeven between the two types of rod occurred in the 39th month after insertion.

The only sensitivity analysis that was conducted varied the rate of device failure (from $0 \%$ used in the base case). First, an $8.8 \%$ failure rate was considered for both arms of the model, taken from the mean device failure for MAGEC rods in the clinical evidence $[7,13,14,16,19]$. Second, a device failure rate of $17.2 \%$ (the highest rate for MAGEC rods in the clinical evidence) was used [16]. Finally, an $8.8 \%$ failure rate in month 0 was considered for both arms of the model, and an additional device failure rate of 1.76 per patient was included at month 13 for conventional rods. The additional device failure at month 13 was misquoted from Yang et al. [41].

The results of the sponsor's univariate sensitivity analysis on the rate of device failure found that the MAGEC system was always cost saving after 6 years. These savings ranged from $£ 8,109$ (breakeven at month 45 ) where device failure occurred $17.2 \%$ of the time in both arms of the model, and £12,984 (breakeven at month 28) where additional device failure, specific to conventional rods, occurred only at month 13 . The results of the sponsor's de novo model led the sponsor to conclude that even with the most conservative assumptions, the MAGEC system is cost saving, compared with conventional growth rods, and cost savings are usually realised during the third year following insertion.

\subsubsection{Critique of Economic Evidence}

A critique of the sponsor's submission was undertaken, which included the literature search for economic evidence, selection of the included studies, the included evidence and the de novo economic model. The EAC attempted to replicate the sponsor's literature search of PubMed for published economic evidence, using the limited description provided, and also undertook its own search in specialist databases indexing economic research (the databases that were searched and the strategies that were used are available from the EAC assessment report [2]). No economic studies additional to the one included by the sponsor [17] were identified.

Given that the economic evidence base consisted of only one study, it was appropriate for the sponsor to build a de novo economic model. The EAC judged that the sponsor's de novo costing model captured the key cost considerations relating to the decision problem. There were, however, limitations relating to the model structure and inputs, which the EAC explored.

The modelled cost inputs were taken from the study by Richards and Nnadi [17], which included 14 patients. The EAC acknowledged that there was a lack of published 
economic evidence available. It sought to verify all inputs used in the model, using data from other sources and expert advice. Other clinical inputs were also validated where possible. After consideration of the components of the model cost inputs, the EAC judged that the sponsor had overestimated the cost of MAGEC rod insertion, MAGEC rod lengthening and conventional rod insertion, and had underestimated the cost of conventional growth rod lengthening and the cost of device failure for both devices.

The EAC contacted clinical experts for advice on the suitability of the structural assumptions that had been made by the sponsor. The key assumption of the sponsor's de novo model was that the clinical efficacy of all outcomes was equivalent between MAGEC and conventional rods. Given the lack of good-quality clinical evidence, it was not possible to prove or disprove this assumption.

Four further structural assumptions were made. First, no adverse events other than device failure were incorporated into the model; however, the EAC deemed that infection rates were well reported within the clinical evidence and should be included within the model. Second, device failure occurred immediately or not at all (except in the final sensitivity analysis in the conventional growth rod arm of the model). Clinical experts advised that device failure occurring only in the same month as rod placement is unlikely. Third, all parameters in the model were identical for both single and dual rods for both the treatment and the comparator, despite large cost variations between single and dual rods. Finally, no discounting was applied within the model. The NICE methods guide states that "A discount rate of $3.5 \%$ (per year), as recommended by HM Treasury, is used to reflect the time value of costs and benefits" [42].

Because of these structural limitations, the EAC replicated the sponsor's model in Microsoft Excel ${ }^{\circledR}$ (making it fully executable) and made four adaptations to the model structure. First, surgical site infections, as reported in the clinical evidence, were included within the model. For conventional growth rods, a monthly infection rate was applied, as these patients undergo both initial insertion surgery and ongoing surgery for distractions. The monthly rate was calculated from the nine studies that reported infection rates in patients with conventional growth rod $[20,24,25,27,28,31-33,37]$. For the MAGEC system, infections were assumed to occur in month 0 - the same month as the initial rod insertion-with the infection rate being calculated from four studies reporting this information $[7,13,14,16]$.

Second, the EAC calculated and applied a monthly rate of device failure over the 6-year time horizon of the model for both the MAGEC system and conventional rods. This was consistent with the advice received from clinical experts, who, on the whole, said that rod breakage could occur at any time. The monthly rate of complete rod breakage was established using the clinical evidence for both the MAGEC system [7, 13, 14, 16] and conventional growth rods [20, 24, 25, 27-33, 37].

Third, the EAC weighted the cost of the device in both arms, according to the proportion of patients receiving single and dual rods within the clinical evidence (65\% dual MAGEC rods and $64 \%$ dual conventional rods). Finally, the EAC discounted costs at a rate of $3.5 \%$ per year, as recommended by HM Treasury [42]. The model input parameters were also updated by the EAC, following validation by clinical experts and against other sources where possible.

Following the amendments made by the EAC to the model structure and inputs, the EAC found that MAGEC rods generated cost savings of $£ 12,077$ per person over the 6 -year time horizon. The cumulative discounted cost over 6 years was $£ 38,242$ for MAGEC rods and $£ 50,319$ for conventional growth rods, with breakeven at month 35 .

Extensive deterministic sensitivity analysis was undertaken around the model inputs. Each model input was varied within a range deemed plausible following consideration of the published evidence and advice from clinical experts. One-way sensitivity analysis showed that the model was robust to changes in most parameters, but it highlighted that the cost and the frequency of distractions of conventional growth rods were the key drivers of the model. This was confirmed by the two-way sensitivity analysis. MAGEC rods would be the more expensive technology if conventional growth rods are distracted less frequently than every 10.2 months if and the majority of distractions are undertaken as outpatient procedures with no complications. Advice from clinical experts suggested that this is unlikely; hence MAGEC rods are likely to be cost saving, compared with conventional rods, when used in the context of the NHS.

\section{NICE Guidance}

\subsection{Provisional Recommendations}

The evidence submitted by the sponsor and the EAC's critique of this evidence was presented to MTAC, who provided draft recommendations relating to MAGEC rods following their meeting in December 2013. These were as follows:

"1.1 The case for adopting the MAGEC system for spinal lengthening in children with scoliosis is supported by the evidence. Using the MAGEC system would avoid repeated surgical procedures for growth rod lengthening. This could reduce complications and 
have other physical and psychological benefits for affected children and their families."

"1.2 The MAGEC system is indicated for use in children with scoliosis, usually aged between 2 and 11 years, who need surgery to correct their spinal curvature, for example when conservative methods such as bracing or casting have failed."

"1.3 Findings from cost modelling estimate that using the MAGEC system is cost saving compared with conventional growth rods from about 3 years after the initial insertion procedure. The estimated cost saving per patient after 6 years is around $£ 12,077$. The cost savings remained robust in sensitivity analyses" [2].

\subsection{Consultation Response}

During the consultation, NICE received 23 comments from eight consultees; consequently, the recommendations were amended to clarify the age group for which the MAGEC system is indicated. This change was also incorporated as part of the technology description, together with an explanation of when the MAGEC system rods should be removed.

A description of the potential increase in cost savings generated by use of the MAGEC system instead of conventional growth rods, through avoidance of spinal cord monitoring, was added to the recommendations. The system impact section of the guidance was expanded to explain that spinal cord monitoring is not needed when lengthening MAGEC rods, but it may be used in conventional rod lengthening. A paragraph was added to the cost evidence and considerations to include findings from revised cost modelling by the EAC to calculate the potential cost savings that would be achieved if spinal cord monitoring is not used during MAGEC lengthening procedures.

Other sections of the guidance were changed to reflect the uncertainty in the proportion of children not requiring interventional treatment for scoliosis, and to provide a more accurate estimate of the number of children who may be eligible for treatment with the MAGEC system in England each year. Minor revisions were made to other sections to improve clarity.

\section{Key Challenges and Learning Points}

The key challenge faced by both the sponsor and the EAC was the lack of robust comparative studies providing evidence on the MAGEC system versus conventional rods. The sponsor's clinical evidence was limited to one comparative study [19] and five case series [7, 13, 14, 16, 18], which had small sample sizes, often included heterogeneous patients and were conducted by different surgeons, with no consistent operating procedures. These were graded as very low quality evidence, with any estimate of the effect size being uncertain. A meta-analysis of these studies was conducted, which was compared with a large conventional growth rod study [20].

The EAC attempted to overcome the limitation in comparative studies by conducting a literature review to identify relevant conventional rod studies and by undertaking meta-analysis on outcomes reported in these studies. Despite this, major limitations existed because of high study heterogeneity (meaning some meta-analysis results could not be used), differences in follow-up durations and variations in the baseline characteristics of the participants. For example, the mean age at surgery for patients who were included in the conventional growth rod studies was 6.4 years, compared with 8.0 years in the MAGEC rod studies. Therefore, the patients in the MAGEC system studies may have had less potential for growth. Statistical techniques cannot readily adjust for such confounding factors.

The sponsor's economic analysis was based upon one single-site cost comparison study [17]; uncertainties existed around the external validity of the modelled cost data. Further, the sponsor's model was not fully executable, meaning that the EAC had to replicate the model in Excel ${ }^{\circledR}$ prior to developing a range of scenarios and sensitivity analysis. In order to fully validate the model, the sources of all assumptions needed to be made clear by the sponsor. Transparent communication between the EAC and the sponsor facilitated this. Advice from clinical experts and use of national cost data enhanced the external validity of the model produced by the EAC.

\section{Conclusion}

The MTEP evaluation process was followed for the MAGEC system. This included a submission of clinical and economic evidence by the sponsor, critical appraisal of this evidence by the EAC, additional work to address remaining uncertainties, drafting of recommendations by MTAC, and a subsequent consultation. Following this process, MTAC judged that the evidence demonstrated sufficient potential benefits of the MAGEC system to patients and the NHS to allow positive recommendations to be made for the device.

Acknowledgments The authors are grateful to the clinical experts and the patient lead identified by NICE, who provided expertise and clinical knowledge, and to staff at Newcastle upon Tyne Hospitals 
NHS Foundation Trust, who provided advice on the disease area and cost information.

Newcastle upon Tyne Hospitals and York Health Economics Consortium are funded by NICE to act as an EAC for the Medical Technologies Evaluation Programme. This summary of the Medical Technology Guidance was produced following publication of the final guidance report. This summary has been reviewed by NICE but has not been externally peer reviewed by Applied Health Economics and Health Policy. Three of the authors (IW, CK and AS) are NHS employees; the NHS has a financial interest in the guidance issued by NICE as a result of this work.

Author contributions The manuscript was prepared by MJ, with contributions from JC, JH, IW, TB, HW, CK and AS. The model was developed by MJ, with advice and quality assurance provided by JC and IW. The statistical analysis was undertaken by TB, with advice from JC. The guarantor for the overall content is AS.

Open Access This article is distributed under the terms of the Creative Commons Attribution Noncommercial License which permits any noncommercial use, distribution, and reproduction in any medium, provided the original author(s) and the source are credited.

\section{References}

1. Campbell B, Campbell M. NICE medical technologies guidance: a novel and rigorous methodology to address a new health technology assessment challenge. Appl Health Econ Health Policy. 2012;10(5):295-7.

2. National Institute for Health and Care Excellence. The MAGEC system for spinal lengthening in children with scoliosis. 2014. http://www.nice.org.uk/guidance/MTG18. Accessed 10 July 2014.

3. Cheung JPY, Samartzis D, Cheung KMC. Focus on management of early onset scoliosis. The British Editorial Society of Bone and Joint Surgery. 2013. http://www.boneandjoint.org.uk/content/ focus/management-early-onset-scoliosis. Accessed 21 March 2014.

4. Scoliosis Association (UK). Scoliosis types. 2010. http://www. sauk.org.uk/about-scoliosis/types-of-scoliosis.html. Accessed 21 March 2014.

5. Scoliosis Research Society. Infantile scoliosis. 2013. http://www. srs.org/professionals/conditions_and_treatment/infantile_scoliosis/ casting.htm. Accessed 20 March 2014.

6. Newson L. Scoliosis (curvature of the spine). In: patient.co.uk. 2012. http://www.patient.co.uk/health/Scoliosis-\%28Curvatureof-the-Spine\%29.htm. Accessed 20 March 2014.

7. Cheung KM, Cheung JP, Samartzis D, et al. Magnetically controlled growing rods for severe spinal curvature in young children: a prospective case series. Lancet. 2012;379(9830):1967-74.

8. Centres for Medicare \& Medicaid Services [CMS]. Growing rods spinal surgery. 2013. https://www.healthnet.com/static/general/ unprotected/pdfs/national/policies/GrowingRodsSpinalSurgery. pdf2013. Accessed 31 Jan 2014.

9. Langensiepen SSO, Sobottke R. Measuring procedures to determine the Cobb angle in idiopathic scoliosis: a systematic review. Eur Spine J. 2013;22(11):2360-71.

10. Scientific Spine. Thoracic spine anatomy. 2011. http://www. scientificspine.com/spinal-anatomy/thoracic-spine-anatomy.html. Accessed 20 March 2014.

11. Akbarnia BAKN, Pawelek JB. Is there a significant increase in thoracic height after growing rod surgery for early onset scoliosis? J Child Orthop. 2011;6(5):387-401.
12. National Institute for Health and Care Excellence. Medical Technologies Evaluation Programme: process guide. 2011. http:// www.nice.org.uk/Media/Default/About/what-we-do/NICE-guidance/ NICE-medical-technologies/Medical-technologies-evaluationprogramme-process-guide.pdf. Accessed 10 July 2014.

13. Akbarnia BA, Cheung K, Noordeen H, Elsebaie H, Yazici M, Dannawi $\mathrm{Z}$, et al. Next generation of growth-sparing techniques: preliminary clinical results of a magnetically controlled growing rod in 14 patients with early-onset scoliosis. Spine. 2013;38(8):665-70.

14. Dannawi Z, Altaf F, Harshavardhana NS, El Sebaie H, Noordeen $H$. Early results of a remotely-operated magnetic growth rod in early-onset scoliosis. Bone Joint J. 2013;95-B(1):75-80.

15. Akbarnia BAMG, Salari $P$, et al. Innovation in growing rod technique: a study of safety and efficacy of a magnetically controlled growing rod in a porcine model. Spine. 2012;37(13): 1109-14.

16. Ellipse Technologies Inc. A retrospective multicenter review of early onset spinal deformity patients that underwent either a primary or revision spinal bracing procedure with the ellipse technologies MAGEC spinal bracing and distraction system. 2013. http://clinicaltrials.gov/show/NCT01716936. Accessed 10 July 2014.

17. Richards J, Nnadi C. Magnetically controlled growing rods versus a conventional growing rod system in the treatment of early onset scoliosis: a cost comparison. 2013. http://www.bsrf.co.uk/ uploads $/ 11 \% 20-\% 20$ Magnetically\%20Controlled\%20Growing\% 20Rods\%20Cost\%20Comparison.pdf Accessed 10 July 2014.

18. Yoon WW, Sedra F, Suken S, et al. Improvement of pulmonary function in children with early onset scoliosis using magnetic growth rods. Spine. 2014;39(15):1196-202.

19. Akbarnia BA, Cheung K, Noordeen $\mathrm{H}$ et al. Traditional growing rods versus magnetically controlled growing rods in early onset scoliosis: a case-matched two year study. 2013. https://www. growingspine.org/news-and-events/growing-spine-study-grouppapers-presented-at-the-imast-and-srs-annual-meeting. Accessed 10 July 2014.

20. Bess S, Akbarnia BA, Thompson GH, Sponseller PD, Shah SA, El Sebaie $\mathrm{H}$, et al. Complications of growing-rod treatment for early-onset scoliosis: analysis of one hundred and forty patients. J Bone Joint Surg Am. 2010;92(15):2533-43.

21. Hadorn D, Baker D, Hodges J, et al. Rating the quality of evidence for clinical practice guidelines. J Clin Epidemiol. 1996;49:749-54.

22. Guyatt GH, Oxman AD, Vist GE. GRADE: an emerging consensus on rating quality of evidence and strength of recommendations. BMJ. 2008;336:924-6.

23. Moher D, Liberati A, Tetzlaff J, Altman D. Preferred reporting items for systematic reviews and meta-analyses: PRISMA statement. BMJ. 2009;339(7716):332.

24. Caniklioglu M, Gokce A, Ozturkmen Y, Gokay NS, Atici Y, Uzumcugil O. Clinical and radiological outcome of the growing rod technique in the management of scoliosis in young children. Acta Orthop Traumatol Turc. 2012;46(5):379-84.

25. Farooq N, Garrido E, Altaf F, Dartnell J, Shah SA, Tucker SK, et al. Minimizing complications with single submuscular growing rods: a review of technique and results on 88 patients with minimum two-year follow-up. Spine. 2010;35(25): 2252-8.

26. Marquez S, Miguel J, Francisco Javier SPG, Alfredo GF, De La Sacristana Nicomedes FBG, Jose QR. Reconstructive surgery in patients with early onset scoliosis (EOS) treated with growing rods. Eur Spine J. 2013;22(1):213-4.

27. McElroy MJ, Sponseller PD, Dattilo JR, Thompson GH, Akbarnia BA, Shah SA, et al. Growing rods for the treatment of scoliosis in children with cerebral palsy: a critical assessment. Spine. 2012;37(24):E1504-10. 
28. Miladi L, Journe A, Mousny M. H3S2 (3 hooks, 2 screws) construct: a simple growing rod technique for early onset scoliosis. Eur Spine J. 2013;22(Suppl 2):S96-105.

29. Pfandlsteiner T, Seidel K, Wimmer C. Growth modulation to continue spinal growth in juvenile scoliosis: 8 year follow up. Eur Spine J. 2012;21(11):2326.

30. Sankar WN, Skaggs DL, Yazici M, Johnston CE 2nd, Shah SA, Javidan $\mathrm{P}$, et al. Lengthening of dual growing rods and the law of diminishing returns. Spine. 2011;36(10):806-9.

31. Schroerlucke SR, Akbarnia BA, Pawelek JB, Salari P, Mundis GM Jr, Yazici M, et al. How does thoracic kyphosis affect patient outcomes in growing rod surgery? Spine. 2012;37(15):1303-9.

32. Watanabe K, Uno K, Suzuki T, Kawakami N, Tsuji T, Yanagida $\mathrm{H}$, et al. Risk factors for complications associated with growingrod surgery for early-onset scoliosis. Spine. 2013;38(8):E464-8.

33. Zhao Y, Qiu G-X, Wang Y-P, Zhang J-G, Shen J-X, Li S-G, et al. Comparison of initial efficacy between single and dual growing rods in treatment of early onset scoliosis. Chin Med J. 2012;125(16):2862-6.

34. Andras L, Joiner E, McCarthy RE, et al. Early onset scoliosis treated with growing rods has a greater increase in T1-S1 length, better Cobb correction and more than twice the number of surgeries compared to Shilla. In: Scoliosis Research Society 48th Annual Meeting and Course; 18-21 September 2013; Lyon.

35. Kabirian N, Akbarnia BA, et al. Deep surgical site infection following growing rod surgery in early onset scoliosis: how does it change the course of treatment? In: Scoliosis Research Society 47th Annual Meeting and Course; 5-8 September 2012; Chicago.
36. Uno K, Suzuki T, Kawakami N, et al. The effect of early fusion at ten years or earlier for early onset scoliosis: comparison between 43 early fusion patients and 39 growing rod patients. In: Scoliosis Research Society 46th Annual Meeting and Course, 7-14 September 2011; Louisville.

37. Wang SZJ, Qiu G, et al. Dual growing rods technique for congenital scoliosis: more than 2 years outcomes. Preliminary results of a single center. Spine. 2012;37(26):E1639-44.

38. Sedra FYW, Shaw M, et al. Treatment of early-onset scoliosis with magnetically driven growth rods: preliminary results with a 1-year follow-up [abstract]. In: 50th Anniversary International Philip Zorab Symposium; 20-21 June 2013; London.

39. Harshavardhana NS, Sedra F, Mahmood B, Altaf F, Noordeen MHH. Surgical results of posterior spinal fusion in adolescent idiopathic scoliosis: correlation of clinico-radiological results with implant density. Eur Spine J. 2012;21:S242-3.

40. Borenstein MHL, Higgins JPT, et al. Introduction to meta-analysis. Chichester: Wiley; 2009.

41. Yang JS, Sponseller PD, Thompson GH, Akbarnia BA, Emans JB, Yazici M, et al. Growing rod fractures: risk factors and opportunities for prevention. Spine. 2011;36(20):1639-44.

42. National Institute for Health and Care Excellence. Medical Technologies Evaluation Programme: methods guide. 2011. http://www. nice.org.uk/aboutnice/whatwedo/aboutmedicaltechnologies/MTEP ProcessGuideAndMethodsGuide.jsp?domedia $=1 \&$ mid=67F0D04A19B9-E0B5-D4E26EBDB76BD2D42011. Accessed 18 June 2014. 